\title{
Migration und Integration in der politischen „Verwaltung“ der Gemeinden
}

\author{
Michael Bommes
}

\begin{abstract}
Kein Funktionsbereich der modernen Gesellschaft ist ohne Organisation denkbar, Organisationen sind in der modernen Gesellschaft ubiquitär: keine Ökonomie ohne Unternehmen, kein Recht ohne Gerichte, keine Politik ohne Parlamente, Parteien, politische Verwaltungen, keine Erziehung ohne Kindergärten, Schulen oder Universitäten, keine Gesundheit ohne Krankenhäuser, Arztpraxen, Rehabilitationseinrichtungen, keine Religion ohne Kirchen oder Vereine. Die Lebensführung in der modernen Gesellschaft ist also organisationsabhängig (Tacke 2001). Der Zugang zu allen Bereichen ist weitgehend organisationsvermittelt, und dies gilt entsprechend auch für Migranten.
\end{abstract}

Es gibt eine reiche Tradition der sozialwissenschaftlichen und historischen Organisationsforschung. Migration spielt hier aber kaum eine Rolle, ebenso wie umgekehrt die Resultate der Organisationsforschung in den mit Migration befassten Wissenschaften bislang kaum aufgegriffen werden. Das muss erstaunen, weil sich die Probleme von Migration und Integration insbesondere in Organisationen stellen - wenn sie sich dort stellen. Denn die Bedeutung von Migration und Integration und deren sozialen Folgen ist nicht selbst-evident. Was Migration und Integration bezeichnen und welche Problemstellungen damit verbunden sind, wird in den Organisationen der Politik, der Ökonomie, des Rechts, der Erziehung, der Gesundheit, des Sports oder der Religion im „Prozess des Organisierens" (Weick 1985) selbst erst festgelegt und bewertet.

"Integration geschieht vor Ort" - so lautet einer der aktuellen Topoi in der politischen Migrations- und Integrationsdiskussion, und in diesem Zusammenhang wird den Kommunen eine zentrale Rolle in der Gestaltung der Integration von Migranten zugewiesen (Bommes 2006). Es gibt aber nur wenig Wissen über die historische Rolle der Kommunen im Migrations- und Integrationsprozess, und zugleich ist ihre systematische Rolle als politische Organisationsform in diesem Prozess kaum wissenschaftlich untersucht worden. ${ }^{1}$

\section{Die historisch-systematische Untersuchung der Rolle von Gemeinden bei der Bildung von Kommunikations- und Beobachtungsformen für Migration}

In welchem Ausmaß und in welcher Weise strukturieren die politische "Verwaltung" von Migrationsprozessen und dabei verwendete rechtsförmige und administrative Unterscheidungs- und Beschreibungsweisen gesellschaftliche Kommunikationsformen über Migration? Gemeinden als politische Gebietskörperschaften kommt unter den politischen Verwaltungen bei der Bildung von Kommunikations- und

1 Beide Desiderata waren der Ausgangspunkt für ein von der VolkswagenStiftung gefördertes Forschungsprojekt zu „Migration und kulturelle Differenz in Gemeinden: Eine historischsystematische Untersuchung", auf dessen Ergebnisse sich der Text stützt. 
Beobachtungsformen für Migration eine besondere Bedeutung zu; denn sie sind im föderalen Staatsaufbau der Bundesrepublik Deutschland zentrale Träger öffentlicher Verwaltung. Seit dem Zweiten Weltkrieg haben sich die verschiedenen Migrationen in jeweils unterschiedlicher Weise auf das Territorium der Bundesrepublik Deutschland verteilt und daher die Gemeinden auf sehr verschiedene Weise tangiert (dazu allgemein Bommes/Rotthoff 1994). Über die Reaktionsformen der kommunalen Verwaltungen als politischen Organisationen auf diese Prozesse ist aber nur wenig bekannt. Das gilt besonders für ihre historische und systematische Rolle bei der Strukturierung und politischen Gestaltung der aus Migration resultierenden Folgeprozesse. ${ }^{2}$

Auch Gemeinden bestehen aus Komplexen von politischen Organisationen wie Gemeindeparlamenten, Verwaltungen und politischen Ämtern. In Organisationen erfolgt die Formulierung und Bearbeitung von Problemen nicht umwelt-, sondern strukturabhängig. Organisationen entwerfen, interpretieren und "gestalten" ihre Umwelten entlang von „cognitive maps“, und Probleme werden auf der Folie vorhandener Lösungen beobachtet und bearbeitet (Weick 1985, Weick/Daft 1984, Weick/Bougon 1986). Organisationsentscheidungen orientieren sich in der Bearbeitung und Absorption von Unsicherheit an eigenen vergangenen und zukünftigen Entscheidungen. ${ }^{3}$

Für die Frage nach der Rolle von Verwaltungen für die Beschreibung der Entwicklung der Migrationsverhältnisse und der damit verbundenen Kommunikationsformen heißt das: Organisationen reagieren auf der Grundlage ihrer Strukturen - Stellen/Personal, Programme, Kommunikationswege - auf Umweltereignisse wie Migration und damit in Zusammenhang gebrachte soziale Folgen, registrieren sie und weisen innen Sinn und Problemformulierungen zu. Bei solchen Zuweisungen bedienen sie sich semantischer Repertoires ${ }^{4}$, die in Organisationen lizenziert sind in dem Sinne, dass darin sozial gültige Beschreibungen der Welt formuliert werden können.

Sinnzuweisungen (Weick 1995) ermöglichen Anschlussentscheidungen über Alternativen wie Zuständigkeit/Nicht-Zuständigkeit, abweisen, vertagen oder überweisen, Leistungen zuteilen oder verweigern, Maßnahmen ergreifen, neue Organisationen oder Organisationsstrukturen wie Stellen oder Programme vorsehen usw. Teil solcher Sinnzuweisungen im Bereich Migration ist die Beobachtung von Kultur und Interkulturalität. Wenn staatliche Administrationen als politische Organisationen bei der „Verwaltung“ von Migration diese als Problemstellung interkultureller Kommunikation konzipieren und in ihre rechtsförmigen und administrativen Entschei-

2 Diese Wissenslücke wird auch durch neuere Publikationen (wie Gestring u. a. 2001, Bukow u. a. 2001, Bukow 2001, Gesemann 2001) nicht geschlossen. Organisation als eigenständige soziale Struktur findet keine systematische Berücksichtigung.

3 Vgl. March/Simon 1958, March 1988, Luhmann 2000; man kann daher Organisationen systematisch als Einrichtungen auffassen, die sich von ihrer Umwelt abgrenzen und als solche reproduzieren durch Entscheidungen, die an Entscheidungen anschließen.

4 Von Semantik ist hier in dem Sinne die Rede, dass sie als Kommunikationsmuster den Horizont sozialer Anschlussmöglichkeiten einschränken; zum Begriff der Semantik im hier verwendeten Sinne vgl. Fuchs 1992, S. $82 \mathrm{ff}$. 
dungsprozesse einbauen 5 , ist dies als Teil des "Prozesses des Organisierens" (Weick 1985) in Verwaltungen, als kontingente Form der Absorption von Unsicherheit zu verstehen. Die aus Migration resultierenden bzw. damit im Zusammenhang stehenden Reaktionsformen in Kommunen sind das Resultat der Strukturbildung kommunaler Organisationen selbst und die Unterschiede zwischen innen das Resultat ihres entscheidungsbasierten Operierens. Das wird im Folgenden am Fall der Handhabung der Arbeitsmigration seit den 1960er Jahren in zwei mittelgroßen Städten gezeigt.

\section{Die „Verwaltung“ der Arbeitsmigration in kommunalen Organisationen: Eine vergleichende Fallstudie}

Die Auswahl zweier mittelgroßer Städte für das Projekt "Migration und kulturelle Differenz in Gemeinden" orientierte sich an externen Kriterien: Der Einbezug zweier Gemeinden aus zwei verschiedenen Bundesländern im Norden und Süden der Bundesrepublik Deutschland sollte gewährleisten, dass Kommunen mit je verschiedenen Gemeindeverfassungen (norddeutsche Ratsverfassung vs. süddeutsche duale Rat-Bürgermeister-Verfassung) einbezogen sind, um den Einfluss formal unterschiedlicher politischer Organisationsstrukturen kontrollieren zu können. Die geographische Nord-Süd-Platzierung erlaubte es zudem, den Einfluss des sog. Nord-Süd-Gefälles und damit zusammenhängende sozialstrukturelle Unterschiede wie die lokale Beschäftigungsstruktur oder die Arbeitslosenzahlen auf die Entscheidungen politischer Verwaltungen in dem hier interessierenden Zusammenhang zu berücksichtigen.

Eine weitergehende inhaltliche Vorab-Festlegung wurde nicht vorgenommen, ausgehend von der Annahme, dass Migration eben, wie erläutert, keinen unmittelbar gegebenen Problemsachverhalt darstellt, auf den politische Organisationen nur reagieren, denn die Wahrnehmung, Formulierung und Bearbeitung von Migration in Organisationen sind nicht umwelt-, sondern strukturabhängig. ${ }^{6}$ Die aus Migration resultierenden bzw. damit im Zusammenhang stehenden Reaktionsformen in Kommunen und gegebenenfalls deren jeweilige Besonderheiten sollten als das Resultat der Strukturbildung kommunaler Organisationen, ihres "normalen“, das heißt im oben genannten Sinne entscheidungsbasierten Operierens untersucht werden. Bei der Auswahl der Gemeinden wurde über die genannten externen Kriterien hinaus nur darauf geachtet, dass sie in ihrer Geschichte von den relevanten Migrationen betroffen waren.

Aus einem solchen Ausgangspunkt resultierten verschiedene Schwierigkeiten für die empirische Untersuchung: Sie musste historisch zurückreichen bis zum Zeit-

5 Normative Anforderungen aus der gesellschaftlichen Umwelt werden nicht übernommen, sondern gegebenenfalls symbolisch aufgegriffen; Meyer/Rowan 1977, Brunsson 1989.

6 Das kann man gut am Fall der illegalen Migration erkennen, die die etablierten Beobachtungseinrichtungen für Migration (polizeiliches Meldewesen, statistische Ämter, Bundesgrenzschutz, Bundesamt für Migration und Flüchtlinge etc.) unterläuft und damit verschiedene Prozesse des sense-making auslöst, die vor allem die von der ungesicherten $\mathrm{Be}$ obachtbarkeit dieser Migration in den Schemata des Meldens und Registrierens ausgehende Verunsicherung bearbeiten. 
punkt der lokal je verschieden einsetzenden Arbeitsmigration und von da an die kommunalen Reaktionen und Verarbeitungsformen in den beteiligten kommunalen Organisationen - Sozialämter, Jugendämter, Schulämter, Allgemeiner sozialer Dienst, Ausländerbehörden, kommunale Parlamente, Arbeitsämter, Wohlfahrtsverbände - untersuchen und analysieren. Ziel war es, die Organisationen zu identifizieren, die kommunal (im doppelten Sinne des Wortes) entscheidende Problemformulierer und Agendasetter werden, und zu fragen, wie sie dazu werden, wie andere Organisationen in einer Kommune an solche Formulierungen anschließen, sie modifizieren und an ihre Entscheidungsprobleme anpassen, etc. Indem sich Kommunen mit Migration wiederkehrend, zunächst diskontinuierlich, schließlich kontinuierlich, befassten, bauten sie in dieser Weise sukzessive Strukturen der Selektivität im Umgang mit Migration auf.

Migranten traten und treten dabei in einer Vielzahl von kommunalen Organisationen als Publikum auf, aber in verschiedener Weise:

a) Sie werden gar nicht als Migranten registriert.

b) Sie werden als Migranten registriert und thematisiert, und es ist organisationsintern zu klären, wie mit ihnen zum Beispiel unter dem Gesichtspunkt des Leistungsbezugs zu verfahren ist. Dies erlaubt die Fortsetzung einer organisationsinternen Routine, ohne dass dies auf andere kommunale Organisationen oder die in der kommunalen Öffentlichkeit etablierten Kategorisierungs- und Beschreibungsweisen ausstrahlen muss.

c) Migranten werden in einer Organisation mit dem Anspruch einer organisationsübergreifenden, die Kommune oder gar „die Gesellschaft“ betreffenden Weise zum Thema gemacht. Es werden ihre öffentliche Relevanz, das Erfordernis von gesonderten Maßnahmen oder von Konsequenzen kommuniziert, die NichtZuständigkeiten oder aber umgekehrt organisatorische Zuständigkeiten neu festschreiben, gegebenenfalls die Umverteilung von Ressourcen durchzusetzen vermögen und andere kommunale Organisationen veranlassen, an diese Thematisierungsweisen und Reklamationen von Zuständigkeiten bzw. Nicht-Zuständigkeiten anzuschließen.

Es ging um die Entstehung von Strukturbildungen, die zu einer übergreifenden, auch andere kommunale Organisationen tangierenden Etablierung von Selektivitäten und Zuständigkeiten führen. Damit etablierten sich Problembeschreibungen für das, was Arbeitsmigration, Aussiedlerzuwanderung oder Asylbewerberzuwanderung kommunal bedeuten, für Lösungen, die für solche Probleme anzustreben sind, und für Mittel zur Erreichung solcher Lösungen, die zugleich Zuständigkeiten bzw. Nicht-Zuständigkeiten bestehender Organisationen reklamieren und Einrichtungen entstehen oder vergehen lassen. Empirisch sichtbar wurde dies daran, dass die Fundstellen in den historischen Daten sukzessive aufeinander Bezug nahmen, dass kommunale Gelegenheiten der Relevanz von Migration durch etablierte Selektivitätsstrukturen geführt, auf organisationsförmige Zuständigkeiten geleitet werden und hier auftauchen.

Die Ordnungsstruktur, die in den Daten zunehmend sichtbar wurde, erwies sich als Resultat genau des Prozesses, der Gegenstand der Forschung war: Die Herstel- 
lung von Ordnung in Organisationen angesichts von uneindeutigen und unbestimmten Sachverhalten, denen im Lichte der Problemstellungen dieser Organisationen ein Sinn verliehen und damit eine Ordnung des Vorkommens in diesen Organisationen selbst zugewiesen wird. Auf der Erhebungsseite manifestiert sich dies darin, dass in den Daten parallel zu dem historischen Prozess der Befassung der Kommunen mit verschiedenen Migrationen immer deutlicher nachvollziehbare Verweisungsstrukturen identifiziert werden können.

Die Art und Weise, in der Migration als Problem in den kommunalen Organisationen der ausgesuchten Städte A und B konzeptualisiert und wie darauf reagiert wird, konnte, wie nachfolgend verdeutlicht werden soll, auf diese Weise aus den administrativen Strukturen beider Städte erklärt werden. Die differenten Strukturvoraussetzungen der Kommunalpolitik sind in beiden Städten in den unterschiedlichen Ratsverfassungen beider Städte, in dem frühzeitigen (A) oder verspäteten (B) Umbau der Sozialverwaltung im Zuge des Ausbaus des Wohlfahrtsstaates in Deutschland und in jeweils stadtspezifischen historischen Entwicklungen verankert. Dies führte in beiden Gemeinden im Falle der angeworbenen Arbeitsmigration und der daran anschließenden Prozesse der Niederlassung zu jeweils ganz anderen organisatorischen Reaktionsbildungen, die auch auf die Art ausstrahlten, in der in beiden Städten im Anschluss daran mit den Zuwanderungen der Aussiedler und Asylbewerber 7 verfahren wurde.

In den 1960er Jahren unterschieden sich Stadt A und Stadt B zunächst nur wenig: Arbeitsmigration war für die kommunale Administration und Politik weitgehend bedeutungslos. Dies findet seinen Niederschlag nicht zuletzt in der sehr sporadischen Datenlage für die 1960er Jahre. Die sog. Gastarbeiter und die daraus resultierenden lokalen Konstellationen tauchten auf dem „Bildschirm“ der Kommunen vor allem als Wohnraum- und Ordnungsproblematik auf. Eine Zuständigkeit über die Feststellung illegaler oder hygienisch bedenklicher Zustände durch die Ordnungs- und Gesundheitsämter hinaus wurde abgelehnt und in die Zuständigkeit der Arbeitgeber und der Arbeitsverwaltung verwiesen.

In der Forschung über die sog. Gastarbeiterwanderung, aber auch in der rückblickenden Selbstbeschreibung von Kommunen wird üblicherweise darauf hingewiesen, dass Ende der 1960er Jahre und zu Beginn der 1970er Jahre der Niederlassungsprozess der Arbeitsmigranten und damit das Verlassen der Wohnheime sowie der Familiennachzug und damit die Verlagerung des Lebensmittelpunktes einsetzten. Dies wird zugleich als der Auslöser für einsetzende Integrationsmaßnahmen in vielen Kommunen angesehen, da sie sich mit den Arbeitsmigranten als neuen Einwohnern konfrontiert sahen (Stang 1982). Eine solche strukturfunktionale Betrachtungsweise findet jedoch bei genauerem Hinsehen keine Bestätigung. In der Stadt $A$ entstand im Verlauf der frühen 1970er Jahre ein sog. „Beratungszentrum für ausländische Arbeitnehmer" (BAA), mit dem die Sozialberatung für die Arbeitsmigranten, bis dahin von den Wohlfahrtsverbänden getragen, in städtische Regie übernommen wurde. Dieses Zentrum entwickelte sich in den 1970er und

\footnotetext{
${ }^{7}$ Auf die hier aber nicht eingegangen werden kann.
} 
1980er Jahren zu der für Arbeitsmigration und ihre Folgen zuständigen und mit Definitionskompetenz ausgestatteten zentralen Organisation in der Kommune.

Diese Einrichtung kann man in ihrer Entstehungsgeschichte, in ihrer Strukturentwicklung und in der Art und Weise, in der sie Arbeitsmigration als Problem konzipiert, nicht angemessen als funktionale Reaktion auf die Strukturfolgen der Arbeitsmigration verstehen. Die Entstehung dieser Einrichtung war vielmehr der Kombination verschiedener "Zufälle" geschuldet, die eine Strukturentwicklung in Gang setzte, die mit ihrer Konsolidierung als angemessene und vorausschauende Reaktion auf die Probleme der Arbeitsmigration nachrationalisiert werden konnte. Zu diesen Zufällen gehörten: eine „anders gemeinte" Ratsanfrage der Opposition im Gemeindeparlament; ein angesichts einer geplanten, aber noch ausstehenden Gründung einer Universität bis dahin unterbeschäftigter kommunaler Hochschulreferent, für den der Auftrag zur Beantwortung dieser Anfrage zu einer Gelegenheit wird, seine sozialwissenschaftliche Beschreibungskompetenz auf das Feld der Arbeitsmigration anzuwenden und die Stadt mit ausgefeilten Problembeschreibungen sowie daraus abgeleiteten umfangreichen Empfehlungen, was organisatorisch zu tun sei, zu versorgen; ein halbherziger Versuch des Oberstadtdirektors, den Personal- und Mittelaufwand für das auf die Empfehlungen des Referenten hin eingerichtete, aber zunächst wenig erfolgreich arbeitende BAA durch Umstrukturierung zu reduzieren; die daraus resultierende Übernahme der Leitung des BAA durch einen Sozialarbeiter, der sein an den neu gegründeten Fachhochschulen erlerntes Wissen dazu nutzte, diese Einrichtung zu der kommunal zuständigen Adresse für alle Problemstellungen auszubauen, die innerhalb anderer kommunaler Verwaltungen mit der Arbeitsmigration und ihren Folgen in Verbindung gebracht wurden. Im Ergebnis führte dies zur Herausbildung einer Organisation, an die schließlich alle Ereignisse vermittelt wurden, die in anderen kommunalen Organisationen wie den Sozial-, Jugend- oder Wohnungsämtern als „Ausländerprobleme“ registriert wurden. Dieses Zentrum entwickelte sich zum kommunal lizenzierten Problemformulierer: Mit den von ihm initiierten kommunalen Programmen und Maßnahmen setzten sich die dort formulierten Beschreibungsweisen der Arbeitsmigration und ihrer Problematik als Problemstellung der Sozialarbeit und der (Sozial-)Pädagogik durch.

Parallel zu dieser innerkommunalen Monopolisierung von Zuständigkeit baute dieses Zentrum um sich herum ein Netzwerk von Vereinen als sog. freien Trägern auf, die eine Reihe von „Maßnahmen“8 in den Bereichen von Pädagogik und Sozialpädagogik, Therapie, Berufsausbildung und sog. Kulturarbeit aufbauten. In die admi-

\footnotetext{
8 Einer "Maßnahme" liegt ein hinreichend einsichtig beschriebenes Problem zugrunde, das der Lösung vermittels der eben zu ergreifenden Maßnahme bedarf, die mit einem quantitativ und zeitlich bestimmbaren Mittelverbrauch verbunden ist. Sog. Maßnahmenträger identifizieren und stellen Probleme dar, deren Lösung sie mittels der Durchführung von Maßnahmen gewährleisten. Für diese beschaffen sie die meisten Mittel durch die Mobilisierung politischer Entscheidungen. Die zeitliche Streckung und Fortführung einer Maßnahme gelingt dann, wenn die Träger einsichtig machen können, dass sie die geeignete Lösung für solche Probleme bereitstellen, die ohne ihr Verschulden nachwachsen (wie im Fall von Kindern und Jugendlichen) oder die ein unvorhersehbares Ausmaß haben, so dass die Fortführung oder gar Ausdehnung einer Maßnahme zwingend erscheint. Diese ...
} 
nistrativen und politischen Entscheidungsprozesse der Kommune hinein dehnte das BAA seinen Einfluss durch die Geschäftsführung des Ausländerbeirates aus. Damit entstand im Verlauf der 1980er Jahre ein pädagogisch sozialarbeiterisches Netzwerk von sog. freien und kommunalen Organisationen, die ihre Kapazitäten aneinander steigerten, indem sie Fördermaßnahmen, Bildungs- und Kulturprogramme, Therapie- und Betreuungsangebote in Abstimmung mit dem BAA entwickelten, anboten und dafür mit Unterstützung des BAA kommunale, Landes- und Bundesfinanzierungen einwarben und beschafften. Das BAA machte in dieser Weise mit den gewonnenen Definitionskompetenzen, der Steigerung seiner kommunalen Haushaltsanteile sowie dem lokalen Aufbau und Management der sog. Ausländerarbeit Migration zu einem kommunal bedeutsamen Thema, das in der Zuständigkeit der Sozialen Arbeit lag. Im Effekt wurde dadurch ein für die Arbeitsmigranten und ihre Familien zuständiger Komplex von Organisationen neben den regulären Einrichtungen der kommunalen Sozialverwaltung aufgebaut. Auf dieser Grundlage besaß das BAA in den späten 1970er und den 1980er Jahren die weitgehend unangefochtene Zentralkompetenz in Sachen Migration und vermochte diese auch kommunalintern gegen konkurrierende Ansprüche, zum Beispiel der Kulturverwaltung, zu behaupten.

Die gewonnene Definitionskompetenz ist dabei im Rückgriff auf eine Semantik ausgestaltet worden, die seit den 1970er Jahren aus den anwachsenden Fortbildungsangeboten der Fachhochschulen, Universitäten und kirchlichen bzw. freien Bildungsträger bezogen werden konnte. In solchen Fortbildungen wurden Arbeitsmigration und ihre Folgeprobleme als Kulturdifferenz, Multikultur und Interkulturalitätsproblematik behandelt sowie die Relevanz und Angemessenheit dieser Konzeptualisierungen vermittelt. Diese Beschreibungsformen wurden und werden als Muster bzw. Kopien bezogen und lokal relevant gemacht in der Ausgestaltung des organisatorischen Komplexes, der die Zuständigkeit für die Arbeitsmigranten und ihre Familien gewonnen hat. Diese Formen rücken die Angemessenheit und Rationalität dieses Komplexes in den Blick und machen den Problembezug, das Lösungspotential und die Legitimität des Mittelverbrauchs der getroffenen und projektierten Maßnahmen einsichtig.

In den 1990er Jahren setzte ein erheblicher Bedeutungsverlust des BAA ein. Dieser war aber keine Folge der abnehmenden Relevanz der Arbeitsmigration, sondern eines internen Strukturproblems, dem Wechsel seiner Leitung. Diese, neu besetzt, missachtete zunehmend wesentliche strukturelle Voraussetzungen des kommunalen Organisationsvertrauens in diese Einrichtung. Mit dem resultierenden Verlust

8 (Fortsetzung) ... Maßnahmengebundenheit begründet einen Modus der Arbeit, in der diese permanent gegen Maßnahmenkonkurrenten in ihrer Alternativlosigkeit vorgeführt wird: Die je ins Auge gefassten oder bearbeiteten Probleme sind dringend und dulden keinen Aufschub, der Mittel- und Personalverbrauch ist daran gemessen eher gering oder auch unverantwortlich niedrig. Mit diesem Darstellungszwang als Mobilisierungsform politischer Entscheidungen ist die Inszenierung der permanenten Außeralltäglichkeit der Arbeit und der geradezu heroischen Leistungen der Beschäftigten verbunden. Dieser Zwang zur kontinuierlichen Erfindung und Konzipierung von Maßnahmen durch Verwaltungen und freie Träger ist für den Bereich der Migration typisch, sofern er als Problemstellung der Sozialen Arbeit und damit der Hilfsbedürftigkeit konzipiert ist (allgemein dazu: Bommes/Scherr 2000). 
seiner Problemdefinitionskompetenz in Sachen Arbeitsmigration wurde der Leiter und damit das BAA zur marginalen Adresse, sofern es um die kommunale $\mathrm{Neu}$ schneidung von Problemen sowie die Rekrutierung und Neuverteilung von Mitteln ging und nicht nur um die Aufrechterhaltung von interorganisatorischen Routinen in der Stadtverwaltung. Mit ihrer Marginalisierung wurde auch die Arbeitsmigration zu einer Restproblemstellung für die Organisation der Sozialen Arbeit.

In der Stadt B wurden die Niederlassung der Arbeitsmigranten und der Familiennachzug seit Ende der 1960er Jahre zunächst vor allem als Wohnraumproblem konzipiert, für das man im Sinne des Subsidiaritätsprinzips insbesondere die Arbeitgeber, das Arbeitsamt, die Gewerkschaften, die Kirchen und die Wohlfahrtsverbände in der Zuständigkeit halten wollte. Über die Behandlung dieser Bau- und Wohnungsproblematik hinaus passierte nicht viel - aus im Wesentlichen zwei Gründen: Im Unterschied zu Stadt A ist Stadt B durch eine Tradition gekennzeichnet, in der Kommunalpolitik von kommunalen Honoratioren an Konsens orientiert getragen und weniger durch die bundesweit etablierte politische Parteienkonkurrenz strukturiert wird. ${ }^{9}$ Auf der Basis der baden-württembergischen Ratsverfassung sind die Verwaltung und ihre politische Leitung, die Bürgermeister und Dezernenten die kommunal zentralen Entscheidungsstellen. Vor diesem Hintergrund schlugen die Strukturveränderungen des Wohlfahrtsstaates seit den 1950er Jahren auf die kommunale Verwaltungsstruktur der Stadt B im Unterschied zu Stadt A erst mit Verzögerung durch. Die Sozial- und Jugendverwaltung und ihr Leitungspersonal standen bis Mitte der 1970er Jahre in der Armen- und Fürsorgetradition. ${ }^{10}$ Sie betrieben kein aktives „Aufsuchen der Probleme in präventiver Absicht“ und sahen in der Problemstellung der Arbeitsmigration keine Aufforderung zur Reklamation von Zuständigkeit, Problemspezifikation und damit verbundener finanzieller und personaler Ressourcenbeanspruchung.

Das Aufgreifen von Problemstellungen der Arbeitsmigration und deren Konzipierung bleibt daher in Stadt B bis in die Gegenwart über die auch als solche in Szene gesetzte persönliche Fürsorge des Verwaltungsdezernenten der Stadt vermittelt. Dieser nimmt sich unter Rückgriff auf Problemformulierungen, wie er sie aus anderen Kommunen, vom Städtetag oder vom Land bezieht, regelmäßig der lokalen Problemlagen der Arbeitsmigration in Stadt B an und macht sie insbesondere im von ihm geleiteten Ausländerbeirat öffentlich zu seiner persönlichen Sache. $\mathrm{Er}$ gewinnt damit kommunal im Modus des politischen Honoratioren, der sich neben seinen professionellen Aufgaben für die Probleme seiner Gemeinde persönlich verantwortlich fühlt, Ehre und Ansehen. Damit werden aber die Arbeitsmigration und die in dieser Weise behandelten Problemlagen gerade nicht zu Strukturfragen der Sozialverwaltung. Der Verwaltungsdezernent irritiert nicht die Nicht-Responsivität der ihm untergeordneten Verwaltung in Sachen Arbeitsmigration, indem er ihre administrative Zuständigkeit reklamiert, sondern ermöglicht ihre Fortschreibung.

9 Dies gilt in der politikwissenschaftlichen Literatur als ein allgemeines Kennzeichen badenwürttembergischer Gemeinden kleiner und mittlerer Größenordnung; s. dazu insbesondere Wehling 1996 und Köser 2000.

10 Zu dieser Tradition vgl. Sachße/Tennstedt 1986, dies. 1988, Stang 1982. 
1980 kam es neben dem Ausländerbeirat zur Einrichtung einer eigens mit spezifischen Problemen der Arbeitsmigration befassten Stelle in der Stadt, einer „Arbeitsstelle für ausländische Bürger“. Sie hatte zunächst den Kindergartenbesuch der Kinder der Arbeitsmigranten sicherzustellen und übernahm zugleich die Geschäftsführung des Ausländerbeirates. Mit der Einrichtung dieser Stelle ging es vor allem um eine Entlastung des Dezernenten durch einen Referenten, dem er die Verantwortung übertragen konnte. Diese Stelle, besetzt mit einem Sozialarbeiter, wurde für die übrigen Verwaltungen zu einer Entlastungsadresse, an die sie solche Fälle verwiesen, die als Ausländer klassifizierbar waren.

Im Übrigen verfolgte die Stadt B eine konsequente Politik der Subsidiarität. Sie förderte einige Maßnahmen freier Träger im Bereich Erziehung, sah aber selbst keine Stellen im Bereich Migration vor. Subsidiarität wirkte als eine Art Primärfilter für alle Maßnahmenvorschläge und Problemformulierungen im Bereich Arbeitsmigration. Nur 1990 wurde eine weitere Stelle für die Bildungsarbeit mit ausländischen Frauen und Mädchen eingerichtet und der Arbeitsstelle zugeordnet. Die Durchdringung des Subsidiaritätsfilters gelang hier im Windschatten der Frauengleichstellungspolitik. Im Übrigen kam es aber mit der Einrichtung der Arbeitsstelle zu keiner mit Stadt A vergleichbaren Herausbildung einer Infrastruktur von internen kommunalen oder freien Organisationen, die auf der Basis von migrationsspezifischen Problembeschreibungen Zuständigkeiten und Ressourcen auf einem selbst definierten Feld beansprucht hätten. Die Arbeitsstelle blieb bis Mitte der 1990er Jahre eine Einrichtung, die ihre semantischen Bemühungen im Bannkreis der Ideosynkrasien des Verwaltungsdezernenten und kommunalen Honoratioren unternahm, denen sie ihre Existenz verdankte und von denen sie verwaltungsintern auch bei der Demonstration der Relevanz der eigenen Tätigkeiten abhängig blieb. Arbeitsmigration wurde bis Mitte der 1990er Jahre vorrangig als ein Problem sozialer Integration und sozialstruktureller Benachteiligung beschrieben. Zu einer Semantik von Kulturdifferenz und -zerrissenheit wurde auffällig Distanz gehalten, da diese für die Verwaltung in Stadt B „linke Subkultur“, von der Sozialdemokratie über die Grünen bis zur Alternativszene, konnotierte.

Mitte der 1990er Jahre, im Gefolge der Oberbürgermeisterwahl zu Beginn der 1990 r Jahre, mit der erstmals nicht ein Verwaltungsvertreter, sondern ein sozialdemokratischer Parteivertreter gewählt wurde, erfuhren die Arbeitsstelle und die Frage der Migration inneradministrativ eine symbolische Aufwertung. Der neue Oberbürgermeister erklärte Stadt B zu einer „offenen und europäischen Stadt“ mit interkultureller Orientierung. Organisationsstrukturell änderte sich dadurch kaum etwas. Die Kontaktstelle und ihr Leiter durften sich damit manchmal mehr im Zentrum des kommunal bedeutsamen Geschehens wähnen. Die organisationsstrukturelle Realität, die weiterhin marginale Position der Stelle in der kommunalen Verwaltung holte inn aber regelmäßig wieder ein. Die Praxis der subsidiären Projektförderung setzte sich, auf der Basis der Bereitstellung von Mitteln des Landes zwischenzeitlich auf erhöhtem Niveau, in den Bereichen der Erziehungs- und Bildungsarbeit mit Frauen und Mädchen fort.

Die Geschichte der Reaktion auf die Arbeitsmigration in den Städten A und B zeigt entsprechend der Ausgangsannahmen, dass eine hohe Pfadabhängigkeit besteht: 
Einmal eingeschlagene Richtungen der Strukturbildung gewinnen schnell Dauerhaftigkeit und legen fest, was dann noch möglich oder wahrscheinlich ist, da alles, was als „Ausländerproblem“ beschrieben wird, in die entsprechenden Strukturkontexte umgeleitet wird. Dies führt im Ergebnis dazu, dass hohe Empfindlichkeiten und Resonanzbereitschaft in den Organisationen, die Zuständigkeiten und Definitions- und Entscheidungskompetenzen in Sachen Arbeitsmigration kumuliert haben, mit struktureller Ignoranz und Nicht-Responsivität in anderen Organisationen einhergehen und sich wechselseitig stützen. Versuche, solche eingespielten Strukturen wieder aufzulösen - wie sie etwa unter dem Stichwort „Öffnung der Regeldienste“ unternommen werden -, lösen dann zum Beispiel Irritation und die Wahrnehmung von Strukturerosion durch Kompetenzüberschreitung oder entsprechenden „Talk“ (Brunsson 1989) aus.

Gleichzeitig strahlen die Beschreibungsmodi von Organisationen, die solche Zuständigkeiten und Definitions- und Entscheidungskompetenzen in Sachen Arbeitsmigration kumuliert haben, und die dabei verwendeten Semantiken auf die übrigen kommunalen Verwaltungen aus. Mit der Überweisung von Problemstellungen der Migration und ihrer sprachlichen Formulierung und Darstellung (im Sinne von Garfinkel/Sacks 1976) an solche Organisationen und ihre fachliche Zuständigkeit scheint zugleich eine innerkommunale Konventionalisierung der Beschreibungssprache einherzugehen; denn sie macht auch für die übrigen Administrationen und ihr Publikum solche Überweisungen einsichtig. Solche Konventionalisierungen erodieren, wenn eine Einrichtung - wie in Stadt A das BAA - Teile ihrer Zuständigkeiten und damit auch ihre Darstellungskompetenzen verfallen lässt. Angesichts ihrer Verankerung in eingespielten Routinen und der „langen Dauer" von Stellen können aber die personellen und programmatischen Strukturen einer solcher Einrichtung nicht durch schnelle Entscheidungen umgebaut oder durch andere innerkommunale Organisationsbildungen vollständig substituiert werden.

\section{Migration als Gegenstand und Resultat kommunalen Organisierens: Ergebnisse und Forschungsfragen}

Im Vergleich der beiden Gemeinden zeigte sich, dass schon vordergründig die organisatorische Strukturbildung in den beiden Kommunen sich nicht als umweltabhängig begreifen lässt. Betrachtet man das Ausmaß der Zuwanderung von Arbeitsmigranten in den 1960er und 1970er Jahren in Stadt A und Stadt B, müsste man umgekehrt zu den gefundenen Ergebnissen erwarten, dass Stadt B eine entsprechende Infrastruktur wie in Stadt A aufgebaut hätte und in Stadt A eine Kontaktstelle wie in Stadt B ausreichend gewesen wäre. ${ }^{11}$ Die Reaktion auf die Arbeitsmigration und die Verarbeitung der davon ausgehenden Irritationen war stattdessen davon abhängig, in welchen Strukturkontexten diese Irritationen zunächst registriert, bearbeitet und in Bestimmtheiten übersetzt wurden. Formale Reaktions-

11 In Stadt A lag der Ausländeranteil 1973 (zum Zeitpunkt des Anwerbestopps) nur etwas über $6 \%$, in Stadt B jedoch bei $11 \%$. Zu Beginn der 1980er Jahre, der Zeit des Familiennachzugs, lag er in Stadt A zwischen 8 und $9 \%$ und in Stadt B zwischen 13 und $14 \%$. Und in den 1990er Jahren liegt er in Stadt A bei etwa $10 \%$ und in Stadt B bei etwa $18 \%$. 
muster, wie sie hier charakteristisch waren, übertrugen sich dann auch auf andere Bereiche der Zuwanderung: In Stadt A findet man eine hohe Bereitschaft zur Organisationsbildung auch in Reaktion auf die noch undeutlichen Probleme der Aussiedler- und Asylbewerberzuwanderung und in Stadt B die fortgesetzte Vermeidung administrativer Zuständigkeit.

Die Differenzen zwischen beiden Gemeinden sind wesentlich in den Strukturunterschieden der Kommunalpolitik, bedingt durch die Ratsverfassungen und die Rolle der politischen Parteien, und in der unterschiedlichen Stellung und Struktur der Kommunalverwaltungen, insbesondere der Sozialadministrationen, verankert. Beides hängt eng miteinander zusammen: In Stadt B treffen wir auf eine stark honoratiorenbasierte, kaum von den Parteien durchdrungene und von der Verwaltung dominierte Kommunalpolitik, die als ein politisches Leitprinzip Subsidiarität pflegt. Parallel dazu und als Ausdruck dessen finden wir einen verzögerten Aus- und Aufbau einer modernisierten, Probleme aufsuchenden und definierenden Sozialverwaltung in Stadt B. Die verschiedenen Migrationen sind daher bis in die Gegenwart nicht als aktiv zu bearbeitendes Problemfeld der Sozialverwaltungen konzipiert worden.

Im Unterschied dazu sind in Stadt A die Kommunalpolitik, die Ratspolitik und die Besetzung der politischen Beamtenstellen in der Verwaltung durchdrungen von den politischen Parteien und ihrem Personal. Dies bedingt eine weit größere Responsivität der Kommunalverwaltung gegenüber dem vom Bund und Land ausgehenden wohlfahrtsstaatlichen Um- und Ausbau in den 1960er und 1970er Jahren. Die Reform der Armenfürsorge im Gefolge des Bundessozialhilfegesetzes von 1961, der Einzug einer erneuerten Sozialplanung und eines Personals, das in den reformierten Hochschulen und Fachhochschulen ausgebildet worden war, in eine im Rahmen der Gebietsreformen der 1970er Jahre neustrukturierte Sozialverwaltung bildeten den Kontext dafür, dass hier Berufsgruppen wie Sozialwissenschaftler und Sozialarbeiter beschäftigt waren, deren erlernte Kompetenzen und Weltdeutungen sie mit der Bereitschaft ausstatteten, unklare Problemlagen wie die Arbeitsmigration zu ergreifen, zu beschreiben und diesbezüglich politische Entscheidungen und Maßnahmen zu initiieren.

Strukturdifferenzen der Kommunalpolitik und -administration erklären die Differenz der organisatorischen Reaktionen auf die Arbeitsmigration und ihre Folgen und die damit verbundenen kommunalen Strukturbildungen. Es besteht aber - wie in ganz Deutschland - trotz aller Unterschiede im Detail eine hohe Übereinstimmung in den semantischen Repertoires der untersuchten Organisationen beider Gemeinden, mit denen Migration und ihre Folgeprobleme in Stadt A und Stadt B beschrieben werden. Politische Verwaltungen erfinden wie andere Organisationen auch ihre semantischen Repertoires, mittels derer sie sich und ihre Umwelt beschreiben, nicht ad hoc. Sie stützen sich einerseits auf Routinen und versorgen sich andererseits mit Varianz durch alltägliches Kopieren sowie durch Beschaffung in Form von Beratungen und Fortbildungen. Die untersuchten Kommunen konnten sich im Fall der Arbeitsmigration nicht auf Routinen stützen. Sie reagierten insbesondere mit Organisationsbildung und mit dem Kopieren von Beschreibungsangeboten. In Stadt B dominierte zunächst der Mechanismus des Kopierens auf der Ebene des politi- 
schen Leitungspersonals, ohne dass dies zu Organisationsbildung führte. Stadt A reagierte vor allem mit Organisationsbildung und überließ es dieser Organisation, Problembeschreibungen zu finden. Diese Organisation, ähnlich wie die 1980 eingerichtete Stelle in Stadt B, versorgte sich und ihr Personal mit Beschreibungsmöglichkeiten für das Problem Migration und seine sozialen Folgen, für mögliche Lösungen und die dazu erforderlichen Mittel durch die Teilnahme an Fort- und Weiterbildungsmaßnahmen, wie sie von Universitäten, Fachhochschulen und Fortbildungsinstituten vorgehalten wurden.

Übereinstimmung in den Repertoires bedeutet keine Übereinstimmung in der Verwendung. Die kontextspezifische Verwendung erlaubt vielmehr die Formulierung unterschiedlich weitreichender Problembeschreibungen und unterschiedlicher Schlüsse hinsichtlich des organisatorisch und finanziell Erforderlichen. Die Verwendung des vergleichbaren Repertoires in Organisationen führt zu durchaus unterschiedlichen Resultaten: Im einen Fall wendet eine Kommune in Reaktion auf die Beschreibung der Migrationsverhältnisse als Kultur- und Interkulturalitätsproblematik dauerhaft erhebliche Mittel auf, mit denen sie kommunale Organisationen und freie Träger ausstattet. Im anderen Fall erkennt sie Interkulturalität als Sachverhalt und damit die Angemessenheit der entsprechenden Semantik durch öffentliche Verwendung an, zieht daraus aber keine organisatorischen Schlüsse und belässt die Migrationsproblematik im Modus der Honoratiorenpolitik weitgehend in der persönlichen Zuständigkeit eines Dezernenten und der ihm zugeordneten Stelle.

Welche Konsequenzen ergeben sich für die Migrationsforschung und die Forschung zu dem damit verbundenen Wandel sozialer und kultureller Strukturen daraus, dass Migrationen und der Modus, in dem sie kommunal als soziale Problemstellungen relevant werden, nicht determiniert sind durch Migrationen als solche, sondern abhängig von der strukturellen Verfassung kommunaler Einrichtungen?

Der Migrationsforschung unterliegt häufig ein expliziter oder impliziter Strukturfunktionalismus, der in folgendem argumentativen Grundmuster seinen Ausdruck findet: Internationale Migrationsbewegungen ziehen soziale Folgen nach sich, die zu grundlegenden sozialstrukturellen und kulturellen Wandlungsprozessen führen. Organisationen der Politik, aber auch der Wirtschaft, des Rechts, der Erziehung, der Wissenschaft oder der Gesundheit reagieren auf diesen Wandel bzw. werden politisch oder wissenschaftlich mit der Forderung konfrontiert, auf diesen Wandel zu reagieren und ihre Strukturen entsprechend anzupassen.

Migration und kulturelle Wandlungsprozesse sind aber, wie gezeigt, keine vorgängigen sozialen Ereignisse, auf die kommunale Organisationen als externe Umweltereignisse reagieren. Das Vorkommen von Migranten in Organisationen und das hier strukturierte Geschehen sind vielmehr selbst Teil des sozialen und kulturellen Wandlungsgeschehens, das mit der Redeweise von internationaler Migration und ihren sozialen Folgen bezeichnet ist. In den Organisationen des politischen Systems so wie in den Organisationen anderer gesellschaftlicher Teilbereiche bilden sich erst die sozialen Strukturen, die als Migration registriert werden. Welche Art Geschehen Migration in einer Kommune und damit in diesem organisierten Teilkomplex des politischen Systems ist, welche kulturellen und sozialen Folgen damit für eine Gemeinde verbunden sind, wird mit der Entstehung von Zuständig- 
keiten und Einrichtungen in den Kommunen, mit der Beschreibung von Problemen, Lösungen und angemessenen Mitteln festgelegt. Was dann geschieht, wird auf der Folie solcher Strukturen registriert und weiterverarbeitet.

Der Modus der Verwendung von Kultur und Interkulturalität als Beobachtungs- und Beschreibungssemantik sowie der Formen der Konstruktion von Fremdheit sind abhängig von ihrer Brauchbarkeit zur Handhabung von organisationsspezifischen Problemstellungen. Konflikte, in denen Zuschreibungsprozesse von Differenz und Fremdheit eine Rolle spielen, werden jedoch in der Forschung theoretisch primär als Auseinandersetzungen zwischen Individuen oder Kollektiven über Ressourcen materieller Bedürfnisbefriedigung und sozialer Anerkennung beschrieben (Bade/ Bommes 1996, Bommes 2004a). Kultur- und Fremdheitsunterscheidungen werden in der Tat relevant gemacht bei dem Versuch der Diskriminierung oder der Positionsverbesserung von Individuen oder Kollektiven. Aber in der modernen Gesellschaft werden die meisten Verteilungen sozialer Ressourcen in Organisationen vorgenommen (Bommes 2004b). Daher müssen solche Unterscheidungen vor allem in Kommunikationsprozessen von Organisationen erfolgreich zur Geltung gebracht werden und Anschluss finden. Im Bezugsrahmen von Organisationen bemisst sich die Anschlussfähigkeit von Unterscheidungen aber an ihrer Weiterverwendbarkeit beim Anfertigen von Entscheidungen. Bei der Untersuchung des Organisierens in Kommunen zeigt sich dann aber, dass die Konstruktion von Kulturdifferenz und Fremdheit selten mit spektakulären Konflikten, sehr oft aber mit der Darstellung von Organisationshandeln, seinen Problemstellungen, Mitteln und Lösungen als angemessen und rational zu tun hat.

Ist Migration in einer Gemeinde aber in der Weise, in der dies hier vorgestellt wurde, angemessen und umfassend beschrieben? Damit ist vor allem die vielfach gestellte Frage angesprochen, ob eine Kommune nicht mehr ist als die Summe ihrer politischen Organisationen. Termini wie Gemeinde, Kommune, Stadt, Gemeinwesen, Stadtgesellschaft und ähnliche referieren die organisatorischen Strukturen einer politischen Gebietskörperschaft und sind zugleich Beschreibungsformen dieser Kommunen als lokale oder örtliche Gemeinschaft. Soziologisch gesehen ist die Lebenswirklichkeit von Migranten wie die aller anderen Individuen durch die Teilnahme an den differenzierten Strukturen von Gesellschaft bestimmt, die mit der lokalen Ganzheitlichkeit, die solche politischen Termini emphatisch artikulieren, verstellt ist.

Die soziale und kulturelle Wirklichkeit, die entsteht, wenn Kommunen als eine Organisationsform des politischen Systems sich mit Migration befassen und dieses Geschehen mit bearbeitbarem Sinn ausstatten, ist im Projekt "Migration und kulturelle Differenz in Gemeinden: Eine historisch-systematische Untersuchung" anhand von zwei Kommunen untersucht worden. Zur kommunalen Wirklichkeit gehört auch die Frage, ob und in welcher Weise Migranten als Teil der kommunalen Gemeinschaft gelten. In welcher Weise solche Thematisierungsformen und die zugehörige kommunale Kommunikation von Gemeinschaft sich in den Organisations- und Selbstpräsentationsformen von Migranten niederschlagen, ist eine zu stellende Frage. Für Kommunen selbst ist gegebenenfalls aber nicht diese, sondern die Frage ausschlaggebend, ob sie in dieser Weise weitermachen können oder ob sich 
etwas ändern muss. Mit anderen Worten: Ein Verständnis der Bedeutung von Migrationen für Kommunen ist zunächst und vor allem durch die Analyse der kommunal organisierten Strukturen ihrer Handhabung zu gewinnen.

Hinter der Verwendung von Ausdrücken wie Stadt, Gemeinde, Kommune verbirgt sich schließlich ein sachliches Problem, das hier dadurch ausgespart wurde, dass diese Ausdrücke synonym zur Bezeichnung des Organisationsverbundes verwendet worden sind, der im politischen System als die Gebietskörperschaft der „Gemeinde" differenziert ist. Geklärt werden musste deshalb nicht, was „Stadt" darüber hinaus bezeichnet - im Wissen um die Untiefen der entsprechenden Diskussionen in der Stadtsoziologie und Sozialgeographie. Die Redeweise von Migration und ihren Folgen bezeichnet eine Vielzahl von Ereignissen in „Städten“, die für Kommunen als politische Organisationsformen bedeutungslos sind: Migranten arbeiten, nehmen Kredite auf, führen Prozesse, spielen Fußball, heiraten, besuchen Schulen usw. In einer Stadt als lokaler Kumulation von parallelen ökonomischen, rechtlichen, politischen, sportlichen, familiären, erzieherischen usw. Geschehnissen betreffen solche Ereignisse in vielerlei Hinsicht Kommunen und ihre Organisationen nicht und kommen in diesen auch nicht vor. Ihr Vorkommen und der Modus ihres Vorkommens sind abhängig von den Strukturen, in denen Organisationen einer Kommune Ereignisse registrieren und ihnen einen Sinn zuweisen.

\section{Literatur}

Bade, Klaus J. / Bommes, Michael

1996 Migration - Ethnizität - Konflikt: Erkenntnisprobleme und Beschreibungsnotstände. Eine Einführung, in: Klaus J. Bade (Hrsg.) Migration - Ethnizität - Konflikt: Systemfragen und Fallstudien (IMIS-Schriften, Bd. 1). Osnabrück: IMIS, S. 11 - 40

Bommes, Michael

2004aÜber die Aussichtslosigkeit ethnischer Konflikte in Deutschland, in: Eckern, Ulrich / Herwartz-Emden, Leonie / Schultze, Rainer-Olaf (Hrsg.), Friedens- und Konfliktforschung in Deutschland - Eine Bestandsaufnahme. Wiesbaden: VS, Verlag für Sozialwissenschaften, S. $155-184$.

2004bZur Bildung von Verteilungsordnungen in der funktional differenzierten Gesellschaft. Erläutert am Beispiel „ethnischer Ungleichheit" von Arbeitsmigranten, in: Thomas Schwinn (Hrsg.), Differenzierung und soziale Ungleichheit. Die zwei Soziologien und ihre Verknüpfung. Frankfurt a. M.: Humanities Online, S. $399-428$

2006 Kommunen als Moderatoren sozialer Integration, in: IMIS-Beiträge, Nr. 28, S. 11 - 24.

Bommes, Michael / Rotthoff, Ulrich

1994 Europäische Migrationsbewegungen im kommunalen Kontext, in: Interne Studien Nr. 100, Hrsg. v. d. Konrad-Adenauer-Stiftung. St. Augustin, S. 93 - 148.

Bommes, Michael / Scherr, Albert

2000 Soziologie der Sozialen Arbeit: Eine Einführung in Formen und Funktionen organisierter Hilfe. Weinheim/München: Juventa-Verlag.

Brunsson, Neils

1989 The Organization of Hypocrisy. Talk, Decisions and Action in Organizations. Chichester: Wiley. 
Bukow, Wolf-Dietrich (Hrsg.)

2001 Auf dem Weg zur Stadtgesellschaft: Die multikulturelle Stadt zwischen globaler Neuorientierung und Restauration. Opladen: Leske + Budrich.

Bukow, Wolf-Dietrich u. a. (Hrsg.)

2001 Die multikulturelle Stadt: Von der Selbstverständlichkeit im städtischen Alltag. Opladen: Leske + Budrich.

Fuchs, Peter

1992 Die Erreichbarkeit der Gesellschaft. Frankfurt a. M.: Suhrkamp.

Garfinkel, Harold / Sacks, Harwey

1976 Über formale Strukturen praktischer Handlungen, in: Weingarten, Elmar / Sack, Fritz I Schenkein, Jim (Hrsg.), Ethnomethodologie: Beiträge zu einer Soziologie des Altagshandelns. Frankfurt a. M.: Suhrkamp, S. 130 - 176.

Gesemann, Frank (Hrsg.)

2001 Migration und Integration in Berlin: Wissenschaftliche Analysen und politische Perspektiven. Opladen: Leske + Budrich.

Gestring, Norbert u. a. (Hrsg.)

2001 Jahrbuch StadtRegion 2001: Schwerpunkt: Einwanderungsstadt. Opladen: Leske + Budrich.

Köser, Helmut

2000 Der Gemeinderat in Baden-Württemberg: Sozialprofil, Rekrutierung, Politikverständnis, in: Pfizer, Theodor / Wehling, Hans-Georg (Hrsg.), Kommunalpolitik in Baden-Württemberg (Schriften zur politischen Landeskunde Baden-Württembergs, Bd. 11), 3. Aufl. Stuttgart: Kohlhammer, S. 153 - 171.

Luhmann, Niklas

2000 Organisation und Entscheidung. Wiesbaden: VS, Verlag für Sozialwissenschaften.

March, James G.

1988 Decisions and Organizations. Oxford: Blackwell.

March, James G. / Simon, Herbert A.

1958 Organizations. New York/London: Wiley.

Meyer, John W. / Rowan, Brian

1977 Institutionalized Organisations: Formal Structure as Myth and Ceremony, in: American Journal of Sociology, 83, H. 2, S. $340-363$.

Sachße, Christoph / Tennstedt, Florian (Hrsg.)

1986 Soziale Sicherheit und Soziale Disziplinierung. Frankfurt a. M.: Suhrkamp.

Sachße, Christoph / Tennstedt, Florian

1988 Geschichte der Armenfürsorge in Deutschland, Bd. 2: Fürsorge und Wohlfahrtspflege 1870 - 1918. Stuttgart: Kohlhammer.

Stang, Heinrich

1982 Ausländerintegration in der kommunalen Praxis: Leitfaden für Kommunalpolitiker und Verwaltungsbehörden. Köln: Deutscher Gemeindeverlag.

Tacke, Veronika (Hrsg.)

2001 Organisation und gesellschaftliche Differenzierung. Wiesbaden: Westdeutscher Verlag. 
Wehling, Hans-Georg

1996 Gemeinden und Kommunalpolitik, in: Baden-Württemberg: Eine politische Landeskunde (Schriften zur politischen Landeskunde Baden-Württembergs, Bd. 1) Stuttgart: Kohlhammer, S. 150 - 171.

Weick, Karl E.

1985 Der Prozess des Organisierens. Frankfurt a. M.: Suhrkamp.

Weick, Karl E. / Daft, Richard

1984 Toward a Model of Organizations as Interpretative Systems, in: Academic Management Review, 9, S. 284 - 295.

Weick, Karl E. / Bougon, Michel G.

1986 Organizations as Cognitive Maps: Charting Ways to Success and Failure, in: Sims, Henry P. / Gioa, Dennis A. (Hrsg.), The Thinking Organization. San Francisco/London: Jossey-Bass Publications, S. 102 - 135.

Weick, Karl E.

1995 Sensemaking in Organizations. Thousand Oaks et al.: Sage. 